\title{
Synthèse des tables rondes
}

\section{Résumé}

Conformément aux recommandations de l'OCDE, les analyses d'impact de la réglementation (AIR) se développent dans de nombreux pays. Elles répondent à des objectifs variés, d'ordre quantitatif (diminuer le poids des normes) ou plus qualitatif (rationaliser le processus de décision). Le contrôle de leur qualité, point faible dans la plupart des pays, relève d'organes qui diffèrent par le statut, le positionnement etl'étendue de leurs pouvoirs. La qualité des AIR dépend aussi de leur capacité à englober l'ensemble des effets, y compris économiques et sociaux, d'une réglementation, à intégrer des scenarii alternatifs et à mener la consultation des parties prenantes. La possibilité d'amender, voire de censurer l'AIR au cours du processus d'examen, comme l'acculturation progressive des pratiques d'évaluation au sein de l'administration et du Parlement, contribue utilement à la rationalisation de la décision publique. Toutefois, l'introduction de clauses de réexamen reste peu fréquente, et l'articulation entre évaluation ex ante et expost encore mal assurée.

(c) 2018 IDMP/Lavoisier SAS. Tous droits réservés

Mots clés : rationalisation, contrôle, coûts, consultation, parlement, Cour des comptes européenne.

\section{Abstract}

Synthesis of the round-table. In accordance with the OECD's recommendations, regulatory impact assessments (RIAs) are gaining ground in many countries. RIAs meet a range of objectives, either quantitative (reducing the regulatory burden) or more qualitative (rationalising decision-making). The quality control of RIAs, which is a weak point in most countries, is entrusted to bodies that are different in terms of their status, position and powers. The quality of an RIA also depends on its capacity to address all impacts of a regulation, including those of an economic or social nature, to allow for alternative scenarios and to include stakeholder consultation. The possibility of amending, or even rejecting, an RIA in the course of analysis, together with the gradual trend towards assessments as part of government and parliamentary culture,

\footnotetext{
*Auteur correspondant : daniele.lamarque@eca.europa.eu

doi:10.3166/pmp.35. 2018.0011 @ 2018 IDMP/Lavoisier SAS - Tous droits réservés.
} 
does much to help with the rationalisation of public decision-making. However, review clauses are rarely used, and little is done to secure a link between ex ante and ex post assessment.

๑) 2018 IDMP/Lavoisier SAS. Tous droits réservés

Keywords: rationalisation, supervision, cost, consultation, parliament, European Court of Auditors.

Les trois tables rondes ont réuni des représentants d'institutions internationales, européennes et nationales, pour débattre des modalités d'exercice de l'étude d'impact, de ses facteurs de succès et de ses effets sur le processus de la décision publique : OCDE, Commission et Parlement européens, Conseil d'État et Autorité environnementale français, Unité Mieux légiférer allemande, Service public de Wallonie, Contrôle fédéral des finances suisse et Cour des comptes européenne (voir liste des participants en annexe).

Les travaux de l'OCDE sur la qualité de la réglementation et la méthodologie de l'étude d'impact, conduits par la Division de la politique réglementaire, montrent les progrès accomplis dans le développement des études ex ante : à la suite de la recommandation de 2012 de l'OCDE, 35 pays se sont désormais engagés par une déclaration politique commune, non contraignante mais explicite, à intégrer l'étude d'impact à un stade précoce du processus législatif et à la conduire selon des principes qui font désormais consensus. Il est acquis en effet que l'étude d'impact vise à rationaliser le processus de décision publique par l'incitation à fixer des objectifs politiques clairs et à démontrer que la législation prévue constitue le meilleur moyen de les atteindre. L'étude d'impact doit être proportionnée à l'importance du sujet, produire une évaluation quantifiée quand c'est possible, prendre en compte l'ensemble des effets attendus (sociaux, économiques, environnementaux) d'une réglementation, et identifier les avantages et les coûts supportés par ses destinataires.

Ce consensus ne saurait toutefois dissimuler une réalité plus complexe, évolutive, variable selon les dispositifs institutionnels des pays, les cultures administratives, les objectifs politiques ou les priorités gouvernementales. Les travaux de l'OCDE comme les audits nationaux consacrés à l'analyse rétrospective de la pratique des études d'impact (ceux de la Cour des comptes européenne sur les études d'impact en 2010 et les évaluations ex post en 2018), celui du Contrôle fédéral des finances suisse en 2017) éclairent les difficultés et les enjeux de l'exercice. L'étude d'impact peut ainsi être investie d'une ambition politique-informer le débat public, encourager la participation des citoyens à la prise de décision publique- ou, plus fréquemment, d'une mission plus opérationnelle - simplifier la réglementation, alléger les coûts supportés par les entreprises et les ménages. L'objectif d'une réglementation plus simple et moins coûteuse peut être traduit en termes quantitatifs : chaque nouvelle norme doit s'accompagner de la suppression d'une, voire de deux ou trois autres normes (one in, one -or two- out). La recherche d'une meilleure qualité du droit peut également se réclamer des principes de la légistique.

Sans méconnaître cette diversité, les participants ont souhaité appréhender l'étude d'impact sous son acception la plus large, c'est-à-dire une évaluation ex ante de l'ensemble des effets, économiques, sociaux et environnementaux, attendus de la législation proposée. Ils se sont accordés pour reconnaître à l'étude d'impact la valeur d'un outil concourant, avec d'autres instruments, à rendre l'action publique plus lisible, plus cohérente et mieux ciblée, visant à fonder le processus décisionnel sur des données factuelles (evidence-based), 
sans pour autant se substituer à la décision politique. L'utilité d'un tel outil est néanmoins intrinsèquement liée à la qualité de ses méthodes, à la fiabilité des données qu'elle utilise et à la validité de son processus, gages de son acceptabilité. Divers organismes de contrôle ont pour mission de vérifier le respect des cadres et des critères établis pour la réalisation des études d'impact.

Un premier débat, qui a porté sur le contrôle et l'évaluation des études d'impact, a donc analysé le fonctionnement des organes chargés de cette évaluation, présents dans près de 9 pays sur 10 de l'OCDE. Cette question est d'autant plus prégnante que l'OCDE, qui élabore et diffuse des guides méthodologiques de l'étude d'impact, signale le contrôle de leur qualité comme le point faible de la plupart des dispositifs. La mise en œuvre des mécanismes de contrôle peut être appréhendée à partir de plusieurs éléments comme le positionnement, les pouvoirs ou les compétences des organes qui en ont la charge.

Un premier questionnement touche en effet le positionnement, interne au gouvernement ou externe, de ces organes de contrôle, avec pour enjeu le difficile équilibre entre l'indépendance de leur statut, qui requiert une certaine distance, et la pertinence de leurs avis pour la décision politique, qui suppose une certaine proximité.

L'indépendance de l'instance de contrôle se trouve confortée si celle-ci se situe à l'extérieur du gouvernement, afin notamment d'éviter le risque d'avis orientés provenant d'agences ou de départements spécialisés, qui apprécient les réglementations à l'aune de leurs propres compétences. Un organe comme le secrétariat général du gouvernement français, placé auprès du Premier ministre et associé au processus d'élaboration de la législation, bien qu'amené à rendre un avis extérieur à l'administration ministérielle qui a élaboré l'évaluation, apparaît intégré à la prise de décision gouvernementale, et ses avis ne sont pas rendus publics.

De plus en plus de pays (comme l'Allemagne, les Pays-Bas et le Royaume-Uni) ont ainsi fait le choix d'un positionnement externe : le Conseil de contrôle des normes allemand, créé en 2006, est composé de dix membres issus des milieux de l'économie, de la recherche et de l'administration; le Regulatory Policy Committee britannique comporte huit experts indépendants issus de la société civile. La Commission européenne a réformé son dispositif avec la création en 2015 d'un Comité d'examen de la réglementation indépendant, mais positionné à l'intérieur de l'institution. En France, le Conseil d'État, organe charnière dans le déroulement de la procédure législative, particulièrement attentif au renforcement de la qualité du droit, auquel il a consacré plusieurs rapports publics, contribue à ce contrôle externe. L'Autorité environnementale française, organisme collégial créé en 2009, ou la Cellule autonome d'avis en développement durable au sein du Service public de la Wallonie, revendiquent la même indépendance. En Suisse, à la suite du rapport très critique effectué par le Contrôle fédéral des finances sur l'évaluation a posteriori des études d'impact, l'hypothèse de confier leur contrôle à la Chancellerie fédérale est à l'étude.

Cette indépendance doit être soutenue par le recours à des méthodes d'évaluation rigoureuses et confortée par la capacité de l'organisme à publier ses avis, que ceux-ci soient contraignants ou non pour le gouvernement : la prétention à éclairer le débat public impose en effet un devoir d'objectivité et de transparence.

Toutes les analyses confirment l'importance de disposer des compétences nécessaires à la réalisation d'une étude d'impact, afin d'évaluer les effets sociaux, économiques ou environnementaux d'une législation, et d'y appliquer les méthodes appropriées. Ces compétences 
sont d'autant plus indispensables que le contenu des études d'impact peut être très variable selon les contextes : certains pays se focalisent sur les coûts qui pèsent sur les entreprises, l'administration ou les citoyens ; l'Allemagne conduit ainsi des analyses très précises sur les charges administratives imposées aux administrations, aux entreprises et aux ménages (les ministères doivent renseigner 37 items), afin d'identifier le coût de conformité, c'est-à-dire l'ensemble des coûts mesurables (et pas seulement les coûts administratifs) entraînés par le respect d'une réglementation pour les services qu'elle concerne. Le Conseil national de contrôle des normes veille à ce que les coûts de conformité des nouvelles réglementations soient indiqués par les ministères de façon compréhensible et calculés conformément à la méthode fixée. Le responsable de l'Unité Mieux légiférer rend compte au ministre d'État auprès de la Chancelière fédérale, coordinateur pour la simplification administrative, chargé de coordonner toutes les mesures réalisées dans les ministères au sein d'un comité des secrétaires d'État qui se réunit chaque mois. Des statistiques sont publiées par ministère, ce qui a un effet incitatif fort; la baisse des coûts administratifs pour l'économie a été estimée à plus de 12 Mds d'euros par an entre 2006 et 2011. Le Royaume-Uni et les Pays-Bas, de même, se focalisent sur la réduction des charges administratives qui pèsent sur les entreprises.

La Commission européenne, la Suisse ou la France, à l'inverse, comme l'OCDE, définissent un champ très large de l'étude d'impact, qui doit couvrir l'ensemble des incidences économiques, sociales ou environnementales d'une législation, et quantifier ses coûts et ses avantages. En Wallonie, la Cellule autonome d'avis en développement durable instituée en 2014 est dotée d'une compétence générale de conseil et d'avis, l'autorisant potentiellement à rendre un avis sur tous les projets du gouvernement et sur tous les types d'impact liés à ces projets ; ces avis sont publics et les ministres auteurs des projets doivent répondre à ses recommandations.

Les organes chargés du contrôle de la qualité des études d'impact peuvent donc rencontrer des difficultés pour évaluer des études d'impact couvrant un champ étendu, que les administrations elles-mêmes ne sont pas toujours outillées pour élaborer. La prééminence de la culture juridique dans l'appareil d'État, observée aussi bien dans les agences suisses que dans l'administration française, apparaît comme un frein à une approche plus économique. Le Conseil d'État français, aux compétences essentiellement juridiques, a ainsi du mal à couvrir toutes les dimensions de ces études. C'est pourquoi son rapport de 2016 préconise une certification technique en amont de sa saisine, confiée à un panel d'experts spécialisés chargé de vérifier le sérieux, la validité et la méthodologie de l'étude d'impact. Les nombreux guides méthodologiques destinés à aider les différents intervenants ne comblent pas, en effet, tous les besoins en la matière. Quant à l'Autorité environnementale française, qui a mis en place un processus abouti d'analyse de tous les projets de la compétence du ministre ayant une incidence sur l'environnement (couvrant également les domaines de l'énergie et des transports), elle confirme le constat récurrent de la mauvaise qualité des analyses socio-économiques, qui ne relèvent pas du même cadre ni des mêmes obligations que les évaluations environnementales.

La consultation des parties prenantes, point clé des études d'impact, vise à associer les citoyens au processus d'élaboration de la décision publique, et à recueillir l'opinion de ses principaux destinataires. La publicité des avis des instances chargées de la supervision des études et le recours à internet facilitent cette consultation : les avis du Conseil d'État ou du Bureau allemand de la réglementation, publics, précèdent le débat parlementaire; ceux de 
l'Autorité environnementale française, publiés dans les 2 heures qui suivent la délibération, précèdent la phase de consultation du public; ceux du Comité d'examen de la Commission, préalables à la transmission du texte au Parlement européen et au Conseil, sont également publics. Depuis 2017, les parties prenantes ont également la possibilité de fournir, en amont, des commentaires sur les «feuilles de route » publiées sur internet et décrivant les initiatives pour lesquelles la Commission envisage de soumettre une proposition législative. La Commission avait toutefois jugé inutile la publication des versions provisoires des rapports d'étude d'impact préconisée par la Cour des comptes européenne à la suite de son audit de 2010. Ces institutions livrent en outre, dans leurs rapports publics, une analyse de leur activité et des insuffisances qu'elles rencontrent, qui permet d'apprécier la maturité du processus de l'analyse d'impact et les améliorations qui s'imposent.

La contribution des destinataires d'une réglementation, ou plus généralement du public, est-elle pour autant effective et complète? Les réponses sont mitigées : si elle est correctement assurée pour les collectivités territoriales françaises, grâce au Conseil national d'évaluation des normes, elle demeure insuffisante pour les entreprises et plus encore pour les citoyens, qui se saisissent peu des informations disponibles sur des législations en examen, destinées à nourrir le débat public.

Un second débat, qui a traversé les trois tables rondes, a porté sur l'impact des évaluations ex ante sur le processus de décision et les relations entre les différents pouvoirs. S'il est reconnu que l'étude d'impact ne se substitue pas à la décision politique, mais vise à l'éclairer, elle n'est pas neutre pour autant. La plupart des avis délivrés par les autorités de supervision sont non contraignants; ils sont toutefois élaborés lors d'un processus en plusieurs étapes qui a pour effet de conduire l'administration à l'origine du projet à modifier, compléter, voire retirer son texte, ou même à réaliser une étude si cette obligation a été omise. Le Comité européen d'examen de la réglementation rend un avis négatif en première lecture sur la moitié de la soixantaine d'études qu'il examine chaque année; après deux avis négatifs, il arrive que des initiatives soient suspendues ou simplement retirées. En France, un seul exemple de retrait a été recensé, en 2009; en revanche, 5 à 10 fois par an, les projets présentés à l'Autorité environnementale sont retirés, parfois pour être présentés, après modifications, un à deux ans plus tard.

Ces itérations ne sont pas inutiles : elles contribuent au contraire très efficacement à renforcer les compétences des administrations, ce qui est sans doute plus important et plus utile qu'un mécanisme de sanction directe. L'amélioration des études environnementales constatée au fil des années signale en effet une acculturation positive au sein des administrations concernées, qui sont les premières responsables de la réalisation des études d'impact.

L'environnement apparaît comme un des domaines privilégiés des évaluations ex ante, mais ce qui marche pour l'environnement est-il avéré dans les autres domaines? Les progrès sont certainement plus ou moins rapides selon les secteurs, les moyens et la culture des administrations concernées. Ils sont accélérés par un effort de formation, et stimulés par la crainte de contentieux, qui conduit les administrations à être plus attentives au respect des procédures.

L'intervention du juge serait-elle de nature à renforcer l'effectivité des avis rendus par les instances de supervision? Le cas français illustre l'échec d'un dispositif juridictionnel qui constituait pourtant l'esprit de la révision constitutionnelle de 2008 : le Conseil constitutionnel a constamment refusé de sanctionner l'absence ou l'insuffisance d'étude d'impact, 
tant au stade initial du processus législatif, où son intervention pouvait avoir du sens, qu'en fin de procédure, où elle n'en aurait plus guère. De même, si le Conseil d'État reconnaît le moyen de l'insuffisance d'étude préalable, par exemple pour les textes applicables aux collectivités territoriales, il n'a jamais sanctionné ce défaut au contentieux.

Le principal levier de l'efficacité des études d'impact paraît donc résider dans un processus d'acculturation progressive consistant à diffuser au sein de la sphère publique les pratiques d'une évaluation, tant ex ante qu'ex post, ouverte à la consultation des parties prenantes et appuyée sur une approche socio-économique et quantifiée de l'action publique.

Quant au principal destinataire des études, le Parlement, son rôle varie selon les dispositifs institutionnels et l'équation politique de chaque pays : il dispose plus souvent de moyens d'expertise propres dans les régimes présidentiels comme celui des États-Unis, tandis que cette expertise se trouve plutôt placée au sein du gouvernement dans les régimes parlementaires. L'exemple européen illustre la convergence des objectifs d'amélioration de la réglementation entre le Conseil et le Parlement, co-législateurs, et la Commission, chargée de l'initiative de la législation, et de son application. Un rapport de la Cour des comptes européenne de 2010 avait souligné cette convergence, qui s'est renforcée avec le programme REFIT de 2012 ( « Pour une réglementation affûtée et performante ») et l'accord interinstitutionnel du 13 avril 2016, par lequel les trois institutions sont convenues que «la législation de l'Union devrait être compréhensible et claire, permettre aux citoyens, aux administrations et aux entreprises de comprendre aisément leurs droits et leurs obligations, prévoir des exigences appropriées en matière d'information, de suivi et d'évaluation, éviter la réglementation excessive et les lourdeurs administratives, et être aisée à mettre en œuvre ». Les études d'impact examinées par le Comité d'examen de la réglementation de la Commission sont transmises au Conseil et au Parlement, qui dispose de sa propre cellule d'analyse, la direction de l'impact et de la valeur ajoutée européenne.

Le Parlement français à l'inverse ne s'est que peu saisi de l'évaluation, dans la discussion des projets de loi que lui transmet le gouvernement, et n'a pas cherché à développer l'évaluation pour ses propositions de lois, qui échappent à l'obligation d'évaluation préalable. L'utilité de l'étude d'impact pour éclairer les débats est encore affaiblie par le fait que des scénarii alternatifs sont rarement étudiés et que les amendements n'y sont pas soumis, alors qu'ils peuvent avoir pour effet de modifier jusqu'à $80 \%$ d'un projet de loi. L'expérience européenne est plus aboutie : la direction chargée des évaluations d'impact et de la valeur ajoutée du Parlement apporte son appui aux députés, en particulier aux rapporteurs et rapporteurs fictifs désignés pour le travail en commission. Les commissions peuvent lui demander une étude complémentaire, notamment, depuis 2005, sur les amendements, faculté assez rarement offerte aux Parlements dans le monde, et que l'OCDE juge très positive. Cette évaluation est toujours totalement réalisée en externe, pour garantir son indépendance.

Le potentiel conflit entre deux légitimités -celle, démocratique, de la représentation parlementaire et celle, technocratique, de l'administration- évoqué lors des débats a paru résolu par le sentiment majoritaire que l'étude d'impact trouve sa principale justification dans l'obligation identique à laquelle sont soumis le pouvoir législatif comme l'exécutif, de rendre compréhensibles pour les citoyens les résultats attendus de l'action publique. Le recours à l'étude d'impact pour les propositions de lois et les amendements significatifs figure d'ailleurs dans les propositions du rapport public de 2016 du Conseil d'État français. 
Il demeure que l'étude d'impact modifie sensiblement le processus de décision, et partant le rôle de chacun de ses acteurs. L'administration à l'origine du projet, responsable des études d'impact ainsi que de la production et de la validation des informations, est tenue d'être plus explicite sur les objectifs et les effets attendus de ses projets de réglementation; elle doit prendre en compte l'impact d'une réglementation sur ses différents destinataires et sur les différents niveaux de gouvernement, qui doivent être consultés à un stade suffisamment précoce. Plusieurs intervenants ont également insisté sur l'utilité de fournir des variantes et des scénarii alternatifs, trop peu souvent élaborés.

L'exemple européen montre que l'étude d'impact nourrit le débat parlementaire, aide à trouver des compromis, promeut un « mieux légiférer en action », et encourage la coopération entre les institutions. Cette coopération est particulièrement nécessaire dans le contexte européen, afin de garantir que les réglementations respectent les principes de subsidiarité et de proportionnalité.

Le rôle du politique s'en trouve-t-il transformé ? Partiellement oui : convié à entrer dans une logique de production d'expertise, il est conduit à davantage objectiver ses choix; on note ainsi la fréquence accrue, dans le discours politique, de la référence au chiffre, sans toutefois que celui-ci n'occulte -ni n'éclaire toujours- le débat : les mêmes chiffres peuvent servir à justifier des thèses opposées, et l'analyse coût-avantages, dont les difficultés et les faiblesses ont été relevées, ne saurait en aucun cas avoir pour objet, ou pour effet, de dicter une solution unique. Le débat sur la production d'études d'impact à l'appui des variantes ou des amendements ne fait qu'illustrer cette émergence de nouveaux paradigmes constitutifs d'une culture politique différente, soumise à des modes de légitimation plus ouverts, plus transparents et -au moins en apparence- plus rationnels dans l'explicitation des objectifs et des résultats attendus de l'action publique. Des réglementations plus claires et plus compréhensibles, élaborées à l'issue d'un processus de consultation, sont aussi de nature à renouveler la relation entre le public et le monde politique, et à restaurer la confiance des citoyens dans leurs institutions.

Le colloque visait enfin à éclairer la place de l'étude d'impact parmi d'autres modes d'analyse et d'aide à la décision.

L'historique de ces mécanismes, aux États-Unis comme en Europe, montre la construction progressive d'un dispositif orienté, à l'origine, vers la simplification administrative et la réduction des charges, évoluant vers sa finalité actuelle, la construction d'une action publique redevable et transparente, telle qu'elle s'exprime aussi bien dans le droit primaire que secondaire. Au sein de la Commission européenne, cette construction s'est traduite par l'élaboration d'un dispositif relativement intégré, passant par l'harmonisation des pratiques des différentes directions générales, et par l'affirmation que le « mieux légiférer » concerne toutes les institutions européennes. Cette harmonisation n'est cependant pas encore totalement achevée, selon les observations de la Cour des comptes européenne qui a consacré deux audits aux études d'impact de la Commission, en 2010 et 2018.

L'articulation entre évaluation ex ante et ex post apparaît de manière évidente comme la condition indispensable d'une meilleure rationalité de l'action publique, matérialisée par un cycle vertueux qui part de la mise à l'agenda politique, se poursuit dans un processus législatif soutenu par l'étude d'impact, débouche ensuite sur la mise en œuvre de l'action publique et soumet enfin celle-ci à une évaluation ex post qui permettra d'ajuster le dispositif initial et d'enclencher un nouveau cycle. Les clauses de réexamen constituent un élément 
essentiel de ce bouclage. Elles existent dans la réglementation européenne, qui comporte ces clauses dans $65 \%$ de ses actes, une proportion qui s'élève à $80 \%$ pour les directives. Les clauses de révision ou de caducité s'appliquent dans un délai maximum de 5 ans pour la législation secondaire britannique, de 3 à 5 ans après l'adoption de la loi allemande.

Toutefois ce type de rationalité est encore trop peu mis en œuvre : on constate plutôt la redondance d'évaluations ex post mal coordonnées, et la faible prise en compte de « l'évaluabilité » des politiques publiques au stade de leur conception. Les évaluations ex post sont insuffisamment prises en compte dans les études d'impact et recouvrent des pratiques diverses dont les méthodes et les standards ne sont pas toujours établis clairement. La Cour des comptes européenne a ainsi relevé l'absence de définition harmonisée et de méthodologie commune des évaluations ex post, qui trahissent encore les différences de cultures des directions générales, selon qu'elles sont exposées au contexte économique ou davantage centrées sur le travail de la Commission. La publication en 2015 de lignes directrices et d'une boîte à outils du « mieux légiférer » constitue une étape importante parmi les efforts entrepris au sein de la Commission pour harmoniser les approches et garantir la qualité des évaluations ex post.

L'urgence enfin, qui caractérise bien souvent la mise à l'agenda (un tiers des projets de réglementation européenne étaient présentés comme prioritaires en 2017), risque de dénaturer l'exercice. En Australie, l'absence d'étude d'impact justifiée par l'urgence est palliée par une obligation d'évaluation ex post dans un délai de deux ans; en Allemagne à l'inverse, tout projet de loi sans exception, même présenté en urgence, doit donner lieu à une étude d'impact.

L'étude d'impact s'inscrit ainsi de manière évidente dans un processus d'acculturation des différents mécanismes de rationalisation de la décision publique, processus facilité par l'existence de cadres et de méthodologies, soutenu par une structure dédiée, affirmé au fil du temps. L'Autorité environnementale française, créée en 2009, a ainsi eu le temps d'inscrire sa pratique dans la durée et dans un champ précis.

L'étude d'impact ne peut s'imposer dans la culture publique sans la combinaison d'un engagement politique et d'incitations administratives joignant « la carotte et le bâton », mêlant des dispositions contraignantes et des dispositifs d'appui, mobilisant actions de formation et soutien méthodologique, facilitant les mises en réseau ou la contribution de professionnels extérieurs. L'étude d'impact demeure en effet, comme tout exercice d'évaluation, susceptible de conduire à des conclusions différentes selon les questions posées et les méthodes employées. Elle invite donc à la rigueur aussi bien qu'à l'humilité.

La confrontation des expériences et des points de vue permet d'éclairer les multiples facettes et les difficultés pratiques de mise en œuvre d'une démarche reconnue comme un élément essentiel de la rationalisation du processus de conception, d'élaboration et de mise en œuvre de la décision publique. Au-delà des spécificités sectorielles, institutionnelles ou culturelles, la convergence des analyses confirme l'intérêt de poursuivre les échanges et l'identification des facteurs de succès. 


\section{Annexe : Participants aux tables rondes}

WOLFGANG HILLER,

Directeur de l'évaluation de l'impact et de la valeur ajoutée européenne, Parlement européen

MARC HOSTERT,

Chef de cabinet du membre luxembourgeois, Cour des comptes européenne

CÉLINE KAUFFMANN,

Responsable adjointe de la Division de la politique réglementaire, OCDE

PHILIPPE LEDENVIC,

Président de l'Autorité environnementale, Conseil général de l'environnement et du développement durable, France

BERNARD NAUDTS,

Membre du Comité d'examen de la réglementation, Commission européenne

STEPHAN NAUNDORF,

Unité Mieux légiférer, Chancellerie fédérale d'Allemagne

JULIEN PIÉRART,

Expert à la Cellule autonome d'avis en développement durable,

Secrétariat général, Service public de Wallonie

ANDREA RENDA,

Head of Regulatory Policy Unit, Centre for European Policies Studies

EMMANUEL SANGRA,

Responsable des évaluations au Contrôle fédéral des finances, Suisse

CHARLES TOUBOUL,

Maître des requêtes au Conseil d'État, ancien rapporteur général adjoint de la section du rapport et des études

JOHN WATSON,

Directeur de la réglementation intelligente et du programme de travail, Secrétariat général de la Commission européenne 


\section{Recommandations aux auteurs pmp@revues.org}

La revue «Politiques et Management Public » publie des articles scientifiques et des notes de lecture portant sur tous les aspects des fonctionnements et des transformations des organisations publiques (Etat, collectivités locales, secteur hospitalier, entreprises publiques, tiers secteur) mais aussi sur les développements des politiques publiques, que celles-ci soient sectorielles ou transversales, européennes, nationales ou locales. Les contributions appartiennent au champ de l'analyse des politiques publiques, $\mathrm{du}$ management public et des administrations publiques et peuvent relever de toutes les disciplines de sciences sociales : science politique, sciences de gestion, sociologie, sciences économiques, histoire, droit... PMP publie également, dans un cadre dédié et limité, des contributions et des débats de praticiens.

Toute proposition devra avoir trait à l'un des deux principaux domaines couverts par la revue ; elle sera soumise, de façon strictement anonyme, à l'avis de trois rapporteurs dont les conclusions seront communiquées aux auteurs dans un délai maximum de trois mois à compter de la réception électronique du document par le secrétariat de rédaction.

Les critères généraux d'acceptation des contributions sont divers : portée théorique, richesse empirique, connaissance et maîtrise du domaine, lisibilité...

Il est instamment demandé aux auteurs de bien vouloir se conformer aux principes ci-dessous pour la publication des articles, des notes de lecture et des contributions des praticiens.

\section{- Procédure de publication des articles}

1. Les auteurs doivent soumettre leur article par voie électronique en se connectant directement sur http://lavoisier.fontismedia.com/pmp ou sur le site de la revue http:// pmp.revuesonline.com.

Les articles doivent être anonymisés.

2. La revue n'accepte que des textes originaux. En soumettant un article pour publication dans celle-ci, le ou les auteurs s'engagent à réserver à la revue l'exclusivité de la publication de leur article pendant une période de trois mois à compter de la date de réception électronique de celui-ci par la rédaction. 
3. Les articles peuvent être soumis en anglais mais devront être traduits pour publication. Leur volume ne doit pas, en principe, dépasser les 50000 caractères espaces compris (hors annexes). Ils doivent être obligatoirement accompagnés de deux résumés d'une dizaine de lignes maximum et d'une liste de cinq mots-clés maximum, les deux en français et en anglais.

4. Les tableaux et graphiques devront êtres assortis de titres clairs et concis, numérotés et présentés sous une forme permettant leur reproduction directe avec indication de la source. Leur référence doit apparaître dans le texte.

5. Les «notes de bas de page» seront les moins nombreuses et les plus concises possible.

6. La bibliographie figure à la fin de l'article. La référence à un ouvrage ou article comportera dans le corps du texte, et entre parenthèses, le nom de l'auteur suivi de l'année de publication et si nécessaire, la page de la citation. Exemple : (Dupont, 2009 : 214). Les références bibliographiques seront présentées et rédigées suivant les normes internationales en vigueur:

- pour un article : DARRIGAND A.,(2002). Modernisation du service public : l'exemple des PTT en 1990 - leçons d'une réforme. Politiques et Management public 20 (4), 135-145. - pour un ouvrage : CROZIER M., (1963). Le phénomène bureaucratique. Seuil, Paris. Il apparaît par ailleurs souhaitable de ne citer dans le texte ou en référence que des ouvrages et articles déjà publiés ; toute référence à un document non publié (working paper par exemple) devra être accompagnée du nom et de l'adresse Internet de la personne ou de l'organisme susceptible de procurer ce document aux lecteurs intéressés.

7. Tous les articles proposés sont soumis pour avis, de façon strictement anonyme, à trois rapporteurs désignés par la rédaction en chef de la revue ; les rapporteurs donneront leur appréciation selon quatre modalités : Accepté tel quel / Accepté avec modifications mineures / A revoir après modifications majeures / Refusé.

A l'issue de la première révision et au retour de l'article ayant intégré les modifications suggérées, les rapporteurs de la première révision seront sollicités pour apporter une deuxième révision. A cette fin, le ou les auteurs sont invités à faire apparaître dans le texte les modifications apportées à leur article ou à joindre une courte lettre les explicitant.

8. Il est de la seule responsabilité des auteurs d'obtenir toute autorisation de reproduction et de «copyright» relatifs aux textes qu'ils pourraient reproduire, sous forme de citation, dans leurs articles. La reproduction d'un texte correspondant à plus de cent mots nécessite une autorisation écrite de l'auteur ou de l'éditeur. 


\section{- Procédures de publication des « Notes de lecture»}

La revue PMP comporte une rubrique "Notes de lecture" qu'elle souhaite vivante et abondamment alimentée.

Trois types de revues de livres peuvent être envisagés : la recension simple (environ 7000 signes espaces compris), la fiche de lecture approfondie, d'un ouvrage ou de plusieurs sur le même thème (environ 15000 signes), enfin la revue exégétique et critique d'un ouvrage classique, notamment étranger et non traduit en français (environ 20000 signes). Elles seront adressées à la Revue sous forme électronique, avec la mention « Notes de lecture ».

\section{- Procédure de publication des « Débats et contributions de praticiens »}

La rubrique est composée :

de communications proposées, à leur initiative ou à la demande de la Revue, par toutes personnes susceptibles d'apporter une contribution originale sur les évolutions intervenues dans le champ de la gestion publique, en France ou à l'étranger ;

d'entretiens avec des responsables d'institutions françaises ou étrangères, dans les domaines du management public et de l'analyse des politiques publiques.

Les propositions d'articles ou d'entretiens devront s'inscrire dans les domaines du management public et de l'analyse des politiques publiques et ne dépasseront pas 20000 signes espaces compris. Elles seront mises en lecture au sein du comité de rédaction. Elles seront adressées à la Revue sous forme électronique, avec la mention Débats et contributions de praticiens. 
